\title{
Analysis of the Change and Trend of Enterprise Production Cost under the Background of Economic Cycle
}

\author{
Xiao Meiling \\ Kunming Metallurgy College, Kunming, 650033, China \\ Xexi123@163.com
}

Keywords: Economic cycle; Nanometer material; Early warning curve; Low carbon economy; MATLAB software.

\begin{abstract}
In the period background of green circular economy, this paper designs the vector model of enterprise cost control using risk discriminate matrix and fits early warning curve of enterprise risk investment using MATLAB software, which get a new analysis method of enterprise cost changes and trend prediction. In order to verify the validity and reliability of the method, this paper takes the enterprise green nano materials research and development costs as the research object, and using MATLAB software carries on processing for the enterprise cost control early warning signal, we can obtain the fitting curve of enterprise cost change risk, which provides technical reference for the research of enterprise production cost.
\end{abstract}

\section{Introduction}

In recent years, there was a kind of new industry in the process of the national economic development, and the industry is the new materials industry [1,2]. With the rapid development of nano technology, new materials industry has become an important part of our national economic growth [3]. Many provincial and municipal governments will also be new material industry as a major pillar industry planning in the future, with the rapid development of enterprises matching cost prediction model should quickly get innovation and development, this paper uses the theory of green low carbon economy to carry on early warning for the risk, which can obtain the corresponding evaluation parameters.

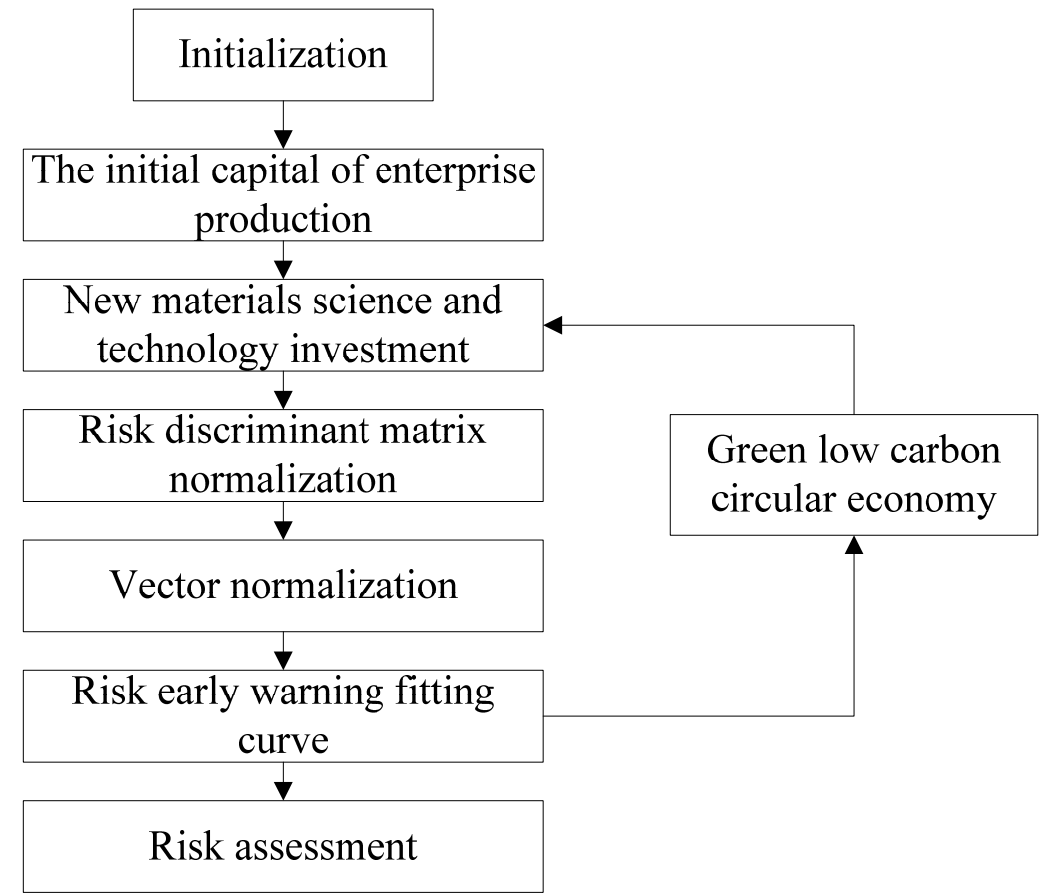

Fig. 1 The process of enterprise production cost evaluation risk

Figure 1 shows the schematic diagram of new materials enterprises production cost assessment process. It can be seen from the chart that the changes trend analysis of the enterprise production cost 
mainly uses the risk judgment rectangular to carry on normalized, risk early warning mainly uses normalized vector to carry on curve fitting, and the calculation and curve fitting of judgment matrix use MATLAB software to achieve [4]. In order to make the model of cost change and forecast analysis to reach optimization, using green low carbon cycle economy theory optimizes the model.

\section{Enterprise Cost Risk Evaluation Vector Normalization Algorithm}

The production enterprises of taking new material as the main development project, the assessment of its cost risk are closely related to the investment of science and technology $[5,6]$. Assuming that the judgment matrix of cost risk $D$ is related to the input indicators of upper technology $F_{1}, F_{2}, \cdots, F_{n}$, the built judgment matrix is shown below:

$$
\begin{array}{cccc}
D & F_{1} & \cdots & F_{n} \\
F_{1} & f_{11} & \cdots & f_{1 n} \\
\vdots & \vdots & \vdots & \vdots \\
F_{n} & f_{n 1} & \cdots & f_{n n}
\end{array} .
$$

Among them, $f_{k l}>0, f_{1 k}=\frac{1}{f_{k l}}$ and $f_{k k}=1(k \neq 1)$, the judgment matrix is normalized available

$$
\bar{a}_{k l}=\frac{a_{k l}}{\sum_{g=1}^{n} a_{h k}}, k, l=1,2, \cdots, n .
$$

For the periodic costs, it can be estimated

$$
\bar{Q}_{i}=\sum_{v=1}^{n} \bar{a}_{k v}, k=1,2, \ldots, n \text {. }
$$

The vector $\bar{Q}=\left[\bar{Q}_{1}, \bar{Q}_{2}, \cdots, \bar{Q}_{n}\right]^{T}$ normalization can obtain

$$
Q=\frac{\bar{Q}_{k}}{\sum_{l=1}^{n} \bar{Q}_{l}}, I=1,2, \cdots, n .
$$

So we can calculate the biggest analysis value of new materials enterprises cost risk.

$$
\alpha_{\max }=\sum_{k=1}^{n} \frac{(D Q)_{l}}{n Q_{l}} \text {. }
$$

For the assessment of cost risk investment, we can be with the help of MATLAB software to calculate, and the cycle curve can be fitted by using MATLAB toolbox, finally we get the risk early warning curve of enterprises cost control, its main procedure is as follows [7,8]:

$\mathrm{A}=\operatorname{sim}($ net,pn);

$\mathrm{E}=\mathrm{A}-\mathrm{tn}$;

$\mathrm{M}=\mathrm{sse}(\mathrm{E})$

$\mathrm{N}=\mathrm{mse}(\mathrm{E})$

Pause

$\mathrm{p} 2 \mathrm{n}=\operatorname{tramnmx}(\mathrm{p} 2, \operatorname{minp}, \operatorname{maxp})$;

$\mathrm{a} 2 \mathrm{n}=\operatorname{sim}($ net, $\mathrm{p} 2 \mathrm{n})$;

$\mathrm{a} 2=\operatorname{postmnmx}(\mathrm{a} 2 \mathrm{n}, \operatorname{mint}, \operatorname{maxt})$ 
echo off

pause

clc

\section{Analysis of the Effect of New Green Environmental Protection Material on Enterprise Cost}

In order to verify the effectiveness and reliability of the mathematical model and algorithm designed in the second part, this paper takes an enterprise green nano materials product research and development costs as the research object, and using the theory of green economy carries on processing for the enterprise cost control early warning signal, in which nano materials are shown in Figure 2.

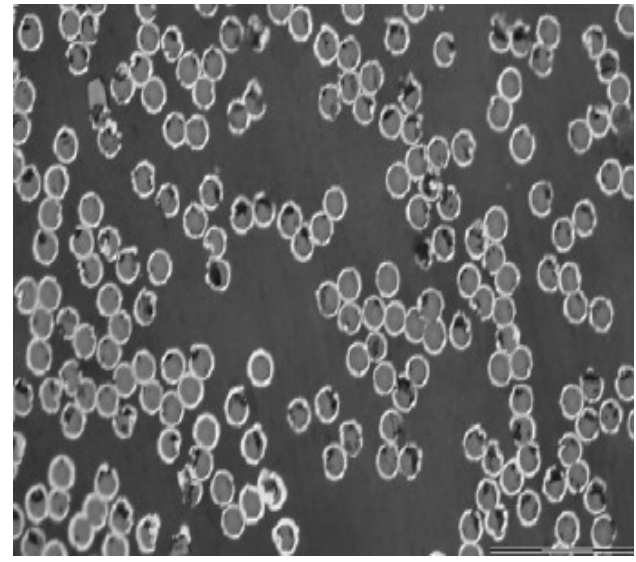

Fig. 2 Schematic diagram of new green nano material preparation

Figure 2 shows the light microscope scanning diagram of new green nano materials. It can be seen from the chart that the alternating multilayer polymer composite electromagnetic shielding materials has good continuity, so that material has good mechanical effect $[9,10]$. The processing cycle of new material is longer, and pre need to invest a lot of technical research and development costs, therefore we need to consider the effects and risks of green circular economy.

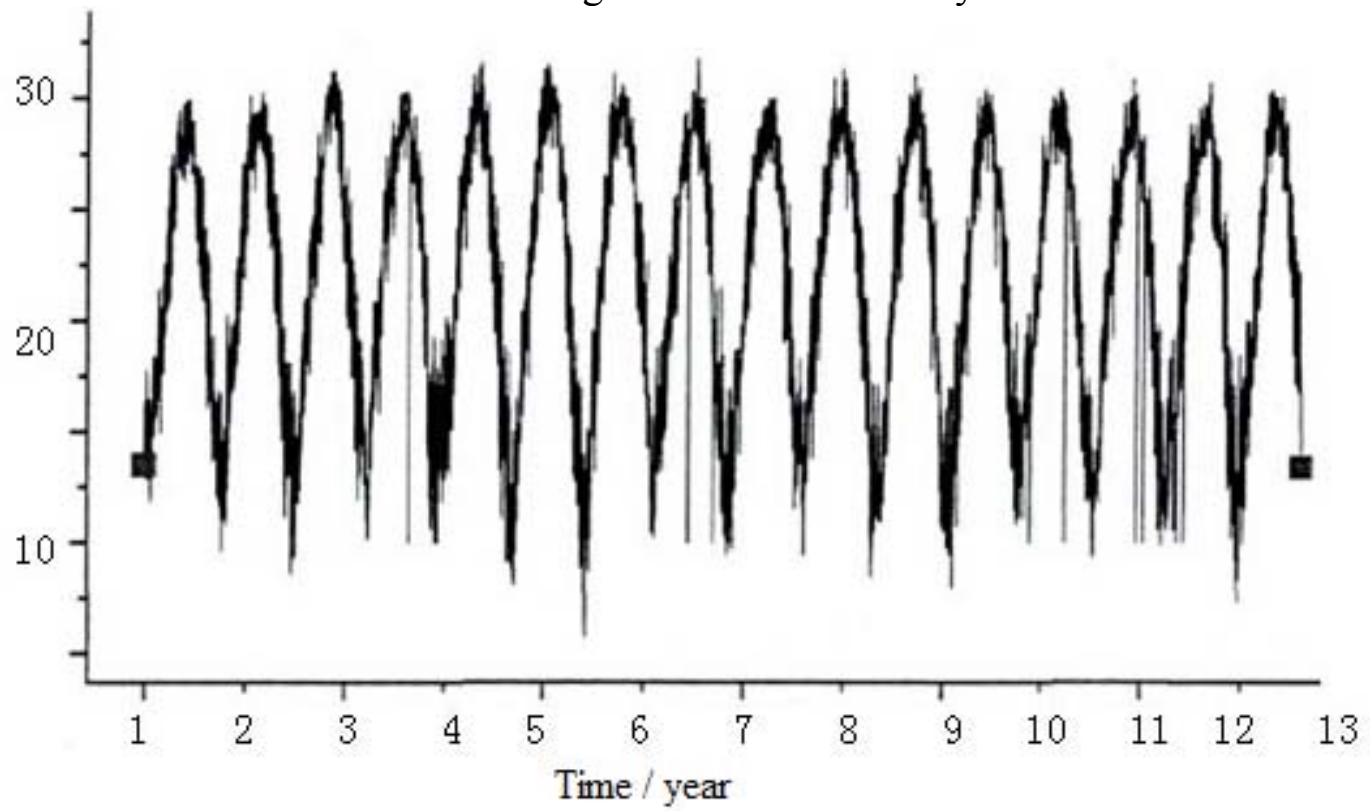

Fig. 3 The analysis curve of enterprise cost control periodic change

Figure 2 shows the analysis curve of enterprise cost control periodic change. It can be seen from the chart that the calculated data shows cyclical fluctuations, and the effect is relatively stable, this is caused by the technical factors of new nano materials in the development process, when the early enterprise development new type materials requires a lot of the cost of technology $[11,12]$. When the 
new materials research and development is successful, the enterprise production cost will reach the highest, and the subsequent input costs will be less and less, it starts a cyclical cycle.

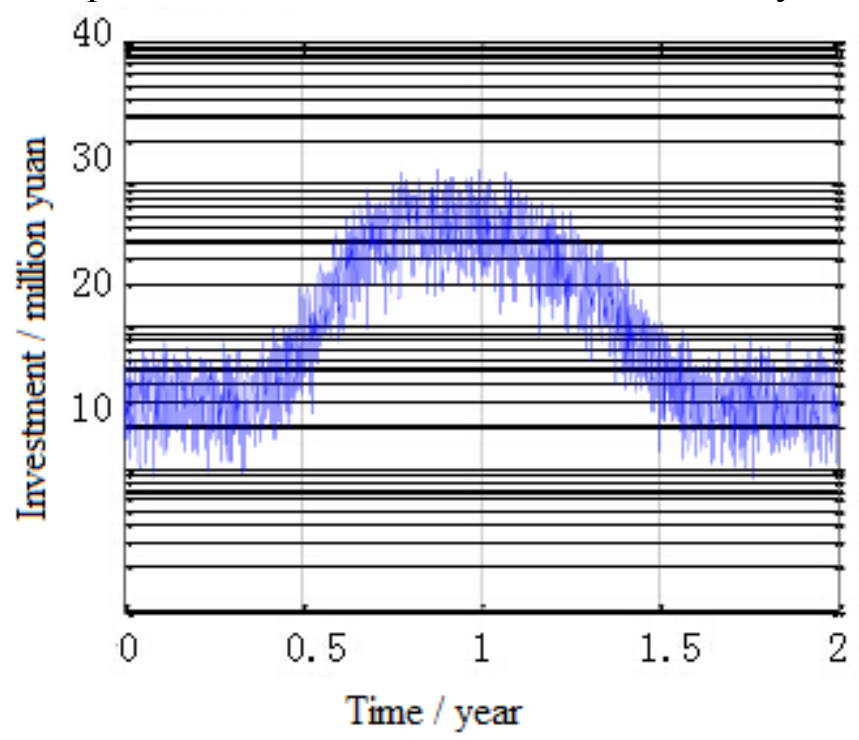

Fig. 4 Risk investment signal amplification curve

Selecting cycle cost investment risk signal caries on analysis, we get the risk early warning of enterprise investment and two years' enterprises control curve, and then after the data are segmented processing we can get the red risk early warning curve.

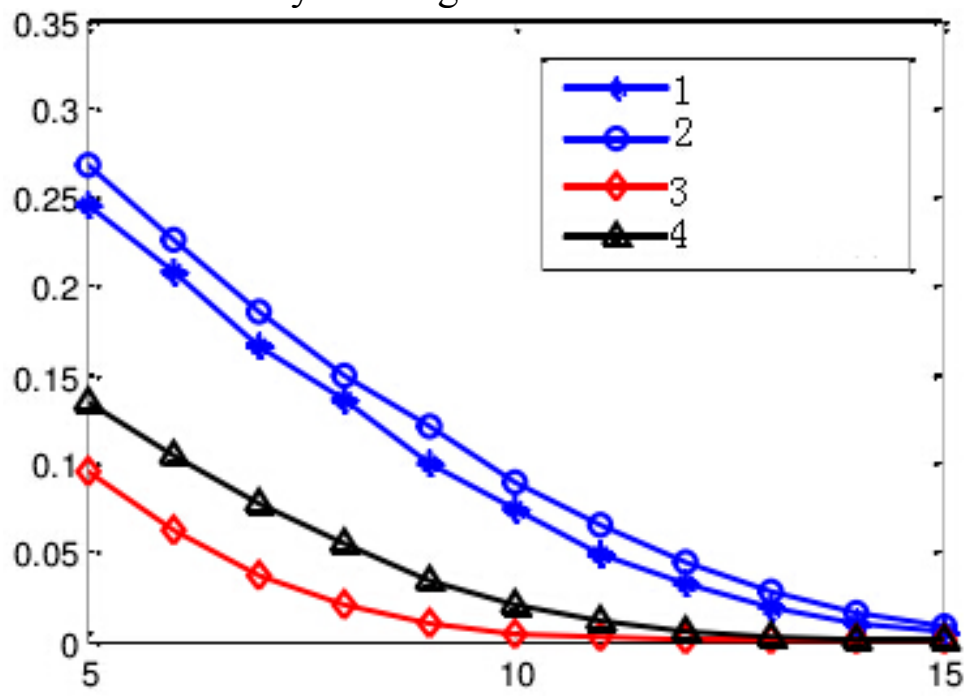

Fig. 5 The curve of enterprise cost red risk early warning

Figure 5 shows the red signal warning curve of the enterprise cost investment risk, in which 1, 2 and 4 curves are the secure curve of risk investment, and the red curve represents that the investment may result in cost risk, therefore we need to be cautious investment in the research and development new nano materials.

\section{Summary}

(1) This paper establishes the risk assessment model of enterprises production costs changes trend prediction and uses the vector normalization algorithm and risk judgment matrix in the model, the algorithm uses the MATLAB software programming, and the final result carries out curve fitting.

(2) Taking the enterprise green nano materials product research and development costs as the research object, this paper carries on calculation for the cost change risk, we can get the MATLAB red warning curve of cost change risk, so as to verify the effectiveness and reliability of the algorithm.

(3) Although cost change discriminant matrix and normalization algorithm effectively solve the cost change warning, it can be applied to more and more fields, such as financing risk evaluation provides technical support and policy advice for the development of enterprises. 


\section{References}

[1]X.T. Sun. A cyclical industry analysis. Journal of North China Electric Power University, 2012(3): 89-90.

[2]D.P. Lin, D.Y. Su. Equity incentive and corporate performance--the new research perspective based on earnings management. Financial research, 2011(9): 56-57.

[3]L. Jiang, X.S. Liu. Study on economic cycle fluctuation and listing corporation cash holdings behavior. Accounting research, 2011(9): 34-35.

[4]Y. Zhao, Z.B. Tan. The electronic commerce, bank credit and small and medium enterprise financing--theory model based on information economics. Economic research, 2012(7): 124-25.

[5]Y. Yu, Y.M. Qian. Macroeconomic shocks and company financing decision in a transition economy: empirical study based on capital structure dynamic adjustment framework. Securities market herald, 2011(10): 45-46.

[6]Chen M.J. The analysis of minor enterprises financing gap--a comparative survey of based on small and medium enterprises in Zhejiang Province. Economic theory and economic management, 2010(11): 105-106.

[7]D.Y. Su, D.P. Lin. Equity incentives, earnings management and corporate governance. Economic research, 2010(11): 56-58.

[8]E.Z. Liu, L.J. Liu. Trade margin and economic cycle coordination-- An empirical study based on China's bilateral trade data. Nankai economic research, 2012(3): 98-99.

[9]J.L. Huang, K.W. Li, D.F. Li.The coordination of Chinese area real economy cycle. World economy, 2011 (9):23-25.

[10]H.F. Cheng, L.J. Cen. Research on FDI, industrial structure and international economic cycle. Research business, 2010 (9): 78-80.

[11]S.C. Liu. Analysis of China's economic growth and fluctuation in 2011 and the "Twelfth Five Year Plan" period. Economic dynamic, 2011 (7): 67-68.

[12]W.D. Zhang. The private placement and earnings management: empirical evidence from China stock market. Management world, 2010 (1): 112-114. 